\title{
Lösungsmöglichkeiten nach fehlgeschlagenen Osteosynthesen am distalen Oberschenkel - Erfahrungen mit dem LIS-System
}

\author{
Hermann Josef Bail, Michael Schütz, Norbert Haas
}

\section{Zusammenfassung}

Die Ergebnisse bei der Versorgung von distalen Femurfrakturen waren mit einer nicht geringen Zahl von Infektionen, Pseudarthrosen und Fehlstellungen belastet. In jüngerer Zeit haben sich die Lösungsmöglichkeiten bei fehlgeschlagenen Osteosynthesen am distalen Femur durch die Einführung minimal invasiv einzubringender winkelstabiler Implantate deutlich erweitert. Die minimal invasiven Operationstechniken, ermöglichen eine stabile Fixation der Fraktur unter größtmöglicher Schonung der ohnehin kompromittierten Weichteile. Kommt es nach primärer Versorgung einer distalen Femurfraktur mit einem "konventionellen" - nicht winkelstabilen - Implantat zum Implantatver- sagen oder zur Fraktur-Redislokation scheint uns daher das Umsteigen auf ein winkelstabiles Implantat (z. B. Femur Less Invasive Stabilization System - LISS) angezeigt. Ebenso können - falls indiziert - biologische Massnahmen wie autologe Knochentransplantation und neuerdings Applikation von Wachstumsfaktoren die Konsolidierung der Fraktur bewirken oder bei einem Implantatwechsel unterstützen. Weitere Möglichkeiten zur Problemlösung bei fehlgeschlagenen Osteosynthesen am distalen Femur sind in Abhängigkeit der Frakturform die Verbundosteosynthese und der frühzeitige alloarthroplastische Gelenkersatz. Unserer Erfahrung nach müssen diese Verfahren bei Anwendung der vorgenannten Techniken nur selten eingesetzt werden.

\section{Einleitung}

Die Schwierigkeiten bei der Versorgung der distalen Femurfrakturen lassen sich schon aufgrund ihrer Ätiopathogenese und Inzidenz vermuten. Die distalen Femurfrakturen entstehen hauptsächlich entweder durch Hochenergietraumata mit entsprechend ausgedehntem Weichteilschaden, häufig im Rahmen von Mehrfachverletzungen, diese dann selbst eine optimale Versorgung und Nachbehandlung der Femurfraktur limitieren oder durch Niedrigenergietraumata, bei denen osteoporotische Frakturen und periprothetische Frakturen dominieren.

Die Therapiekonzepte sind mit konservativer und operativer Therapie, wie extraund intramedulläre Verfahren und primärer alloarthroplastischer Ersatz, viel-

OP-JOURNAL 2004; 20: 158-165

(c) Georg Thieme Verlag KG Stuttgart · New York gestaltig [4]. Die Ergebnisse bei der Versorgung von distalen Femurfrakturen waren mit einer nicht geringen Zahl von Infektionen, Pseudarthrosen und Fehlstellungen belastet. Wegen dieser Komplikationen wurde bis in die 70erJahre von einer routinemäßigen operativen Versorgung dieser Frakturen abgeraten [10]. Erst als geeignete Implantate zur Verfügung standen und gute Ergebnisse publiziert wurden, wandelte sich die Behandlung zur operativen Therapie $[2,8,11]$. Die Plattenosteosynthese wurde bei distalen Femurfrakturen vielfach angewendet, die Wahl des extramedullären Implantates war von der Fraktur abhängig.

Bei den A-Frakturen, C1-Frakturen und einfachen C2-Frakturen wurden partiell winkelstabile Implantate wie die Winkelplatte oder die DCS (Dynamic Condylar Screw) eingesetzt, die bei Trümmerfrakturen nicht geeignet waren $[6,8]$. Bei C3-Frakturen konnte bis weit in die
90er-Jahre hinein nur ein nicht winkelstabiles Implantat wie die Burri-Abstützplatte genutzt werden, die mit Problemen wie der geringen Haltekraft der distalen Schrauben, dem Abkippen der Schrauben und dem Varuskollaps behaftet war $[1,3,6]$. Die Plattenosteosynthese am distalen Femur ging mit zum Teil hohen Komplikationsraten einher. In der neueren Literatur finden sich bis zu 25\% Infektionen bei der operativen Versorgung von distalen Femurfrakturen mit einer Kondylenplatte und Pseudarthroseraten von bis zu $7 \%[4,12]$.

Im Laufe der weiteren Entwicklung wurden Implantatmaterialien, Repositionstechniken, Techniken der Implantatplatzierung und die Implantate selbst verändert. Speziell bei der extramedullären Osteosynthese, bei der die Wiederentdeckung und Einführung der Winkelstabilität im Vordergrund stand, haben sich interessante Entwicklungen vollzogen. So wurde in der Problemregion distales Femur das erste minimalinvasive winkelstabile Fixateur-interne-System eingesetzt, das so genannte LIS-System (Less Invasive Stabilization System). In den unten stehenden Fallbeispielen werden sowohl Problemlösungen mittels LISS nach fehlgeschlagenen Osteosynthesen mit „herkömmlichen“ Implantaten beschrieben als auch auf auftretende Probleme nach der Osteosynthese mit LISS am distalen Femur und deren Lösung eingegangen.

\section{Hauptteil}

\section{Verzögerte Heilung und Pseudarthrosen}

Die distale Region (AO 32 distales Schaftdrittel, und 33 alle) ist die beim Femur am geringsten durch Weichteile bedeckte Lokalisation. Vor allem durch das Frakturtrauma - insbesondere bei offenen Frakturen - wird eine Schädigung des ohnehin dünnen Weichteilmantels hervorgerufen. Besonders bei intraartikulären Trümmerfrakturen bedingt die Anforde- 

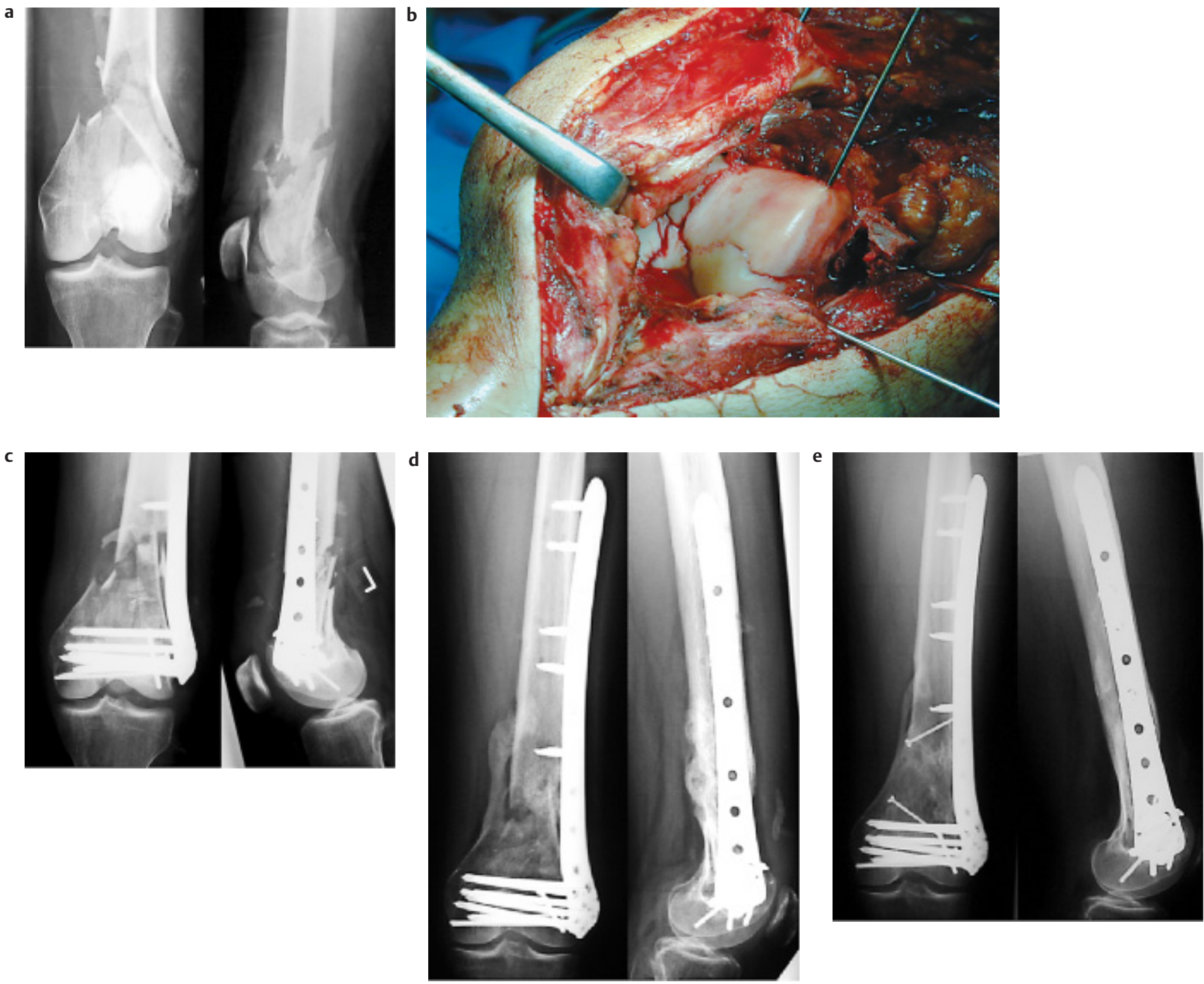

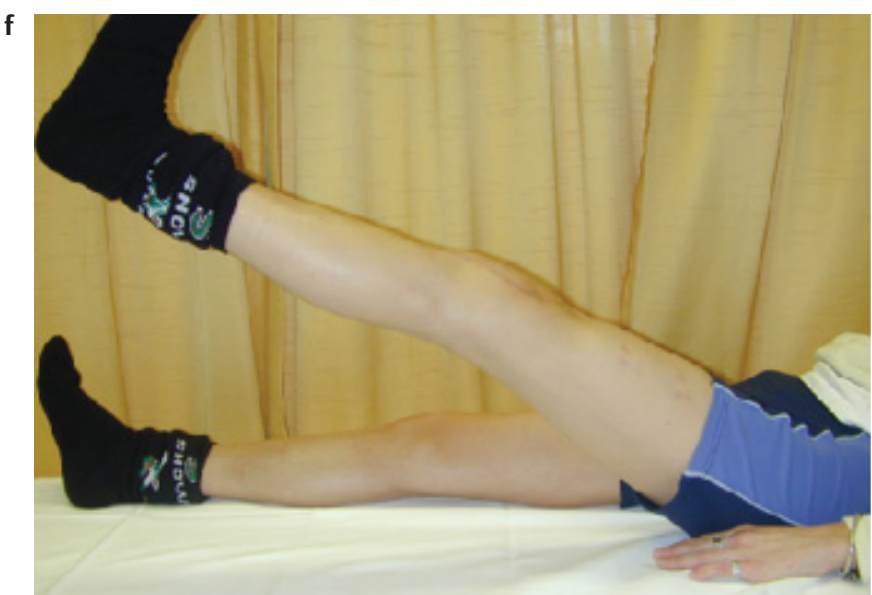

Abb. 1 Pat. 20 Jahre, 33 C 3.2, III-gradig offen. (a) präoperativ (b) intraoperativer Befund nach Rekonstruktion der Gelenkfläche (c) postoperatives Röntgenbild, medialer Knochendefekt erkennbar (d) 4 Monate

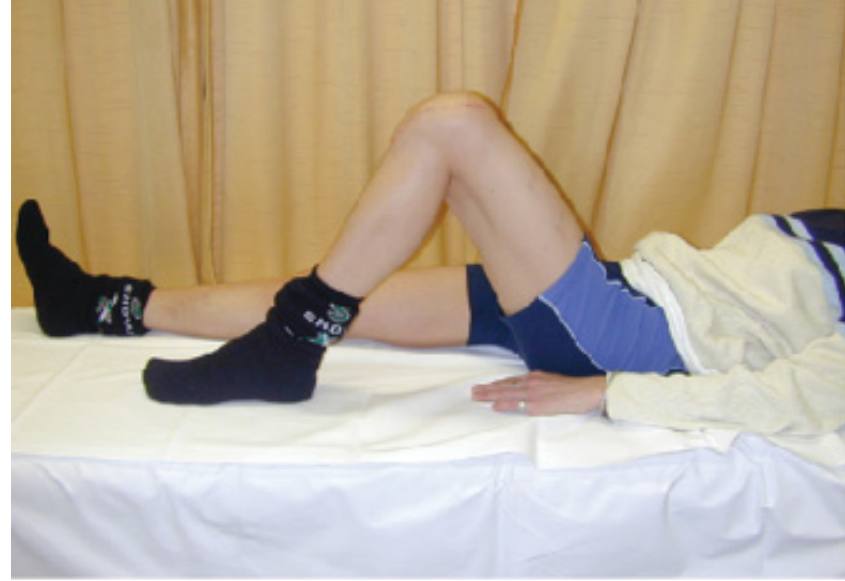

postoperativ (e) 12 Monate nach dem Unfall, Z.n. Transplantation eines autologen Beckenkammspanes welcher mit 2 3,5 mm Kortikalisschrauben befestigt wurde (f) klinischer Befund nach 12 Monaten. 
rung einer anatomischen Wiederherstellung der Gelenkkongruenz eine weitere Kompromittierung des Weichteilmantels. Neben Fragmentdevaskularisation und Periost-Stripping sind diese Faktoren ursächlich für die zum Teil beschriebenen hohen Raten an verzögerter Frakturheilung bei distalen Femurfrakturen. Operative Strategien, welche diesen Faktoren Rechnung tragen, sind schon bei der initialen Versorgung dieser Frakturen anzuwenden. Um so mehr müssen sie jedoch beim Auftreten von Problemen im Verlauf der Frakturheilung berücksichtigt werden: Die Verzögerung der Frakturheilung zeigt an, dass eine oder mehrere der Bedingungen ungestörter Verheilung nicht erfüllt sind.

Im Wesentlichen liegen die Ursachen auf mechanischem und/oder biologischem Gebiet. Für die Strategie des weiteren Vorgehens gilt es zu erkennen, „woran es liegt".

\section{Biologische Ursachen}

Fallbeispiel 1

33 C 3.2, III B offen. Der 20-jährige, polytraumatisierte Patient hat sich diese Fraktur im Rahmen eines Überrolltraumas als Motorradfahrer zugezogen. Nach initialer Transfixation des Kniegelenkes mittels AO-Fixateur erfolgt die definitive Stabilisierung nach Konsolidierung der Weichteile. Bei diesem Fallbeispiel sind folgende, die Frakturheilung kompromittierenden Faktoren vorliegend: 1. Hochenergietrauma ursächlich für die Fraktur, 2. Vorliegen einer offenen Fraktur mit ausgedehntem Knochenverlust medial (Abb.1 a), 3. Darstellung der Gelenkfläche für anatomische Rekonstruktion nötig mit weiterem Weichteilschaden (Abb. 1 b). Nach der Versorgung mit einem Femur-LISS (Less Invasive Stabilization System) verbleibt bei korrekter Rekonstruktion von Länge und Achse des Femurs ein medialer Knochendefekt (Abb.1 c). Das Implantat ist auch nach 16 Wochen stabil ohne Lockerungszeichen (Abb.1d). Es fehlt die mediale Überbrückung, Vollbelastung konnte bis dahin nicht erlaubt werden. Zur Überbrückung des medialen Knochendefektes erfolgte die Implantation eines autologen trikortikalen Beckenkammspans und eine autologe Spongiosatransplantation (Abb.1e). Die Abbildung (Abb.1f) zeigt das klinische Ergebnis mit einer Beweglichkeit von Streckung/Beugung 0/10/110.
Mechanische und biologische Ursachen

Fallbeispiel 2

Der Patient hatte im Rahmen eines Sturzes eine distale Femurfraktur 33 C 2.2 erlitten. Der Patient hatte nach einem Polytrauma 3 Jahre zuvor bei einer Femurschaftfraktur nachfolgend eine Osteomyelitis im diaphsären Bereich des Femurs entwickelt, welche mit einmaligem Fisteldebridement und Markraumaufbohrung bisher saniert werden konnte. Als Begleiterkrankung bringt der Patient einen insulinpflichtigen Diabetes mellitus und einen Nikotinabusus mit. Die aktuelle distale Femurfraktur wurde zu-
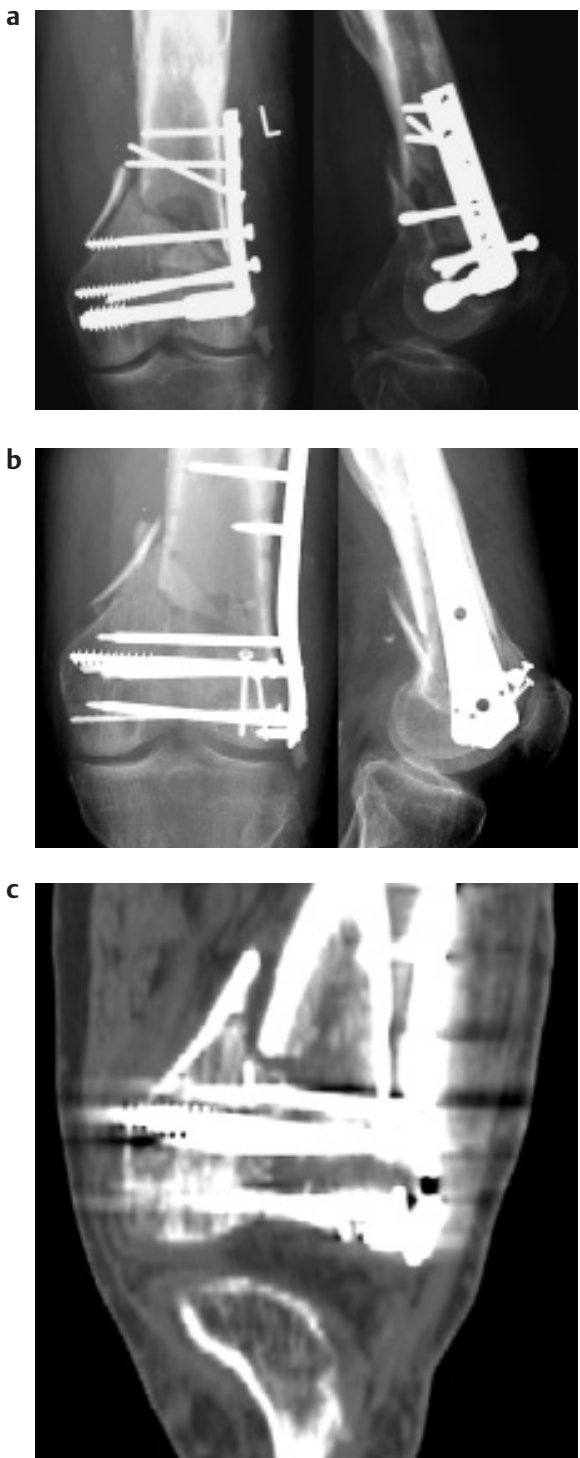

nächst auswärts mit einer dynamischen Kondylenschraube (DCS) versorgt. Es kam 4 Wochen später zu einer Lockerung der diaphysären Schrauben mit Instabilität der Fraktur (Abb.2a). Es erfolgte die Reosteosynthese mit einem Femur-LISS (Abb.2b). Unter der Annahme einer instabilitätsbedingten Heilungsverzögerung wurden zu diesem Zeitpunkt keine biologischen Maßnahmen (z.B. Spongiosaplastik) ergriffen. Im Verlauf konnte auch nach 5 Monaten keine relevante Kallusbildung gesehen werden (Abb. 2c). So kam es trotz fortwährender Teilbelastung an Gehstützen zu einem Schraubenbruch und zur Dislokation des distalen Fragmentes nach dorsal (Abb. 2d). Zwischen-
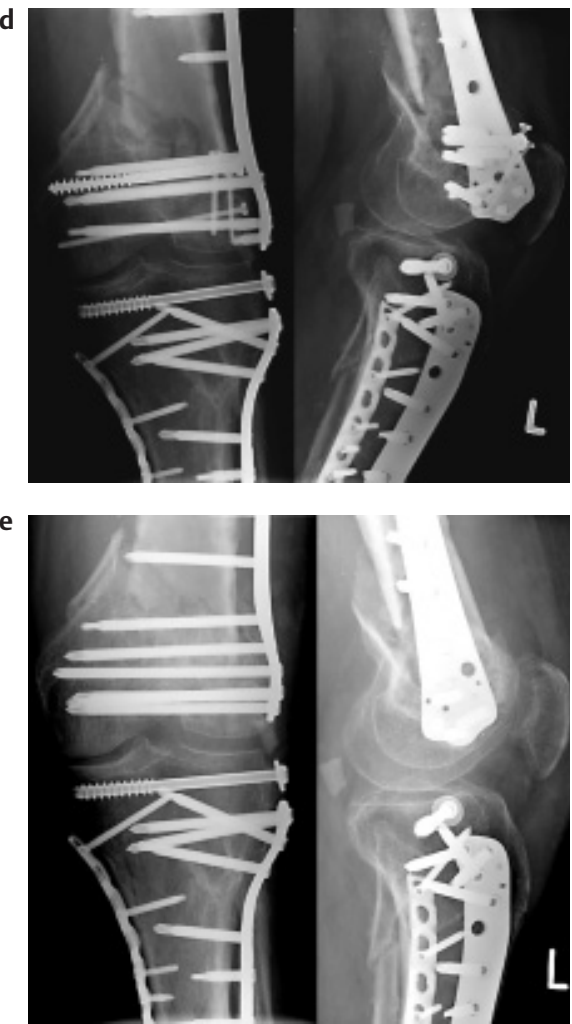

Abb. 2 Pat. 62 Jahre, primär auswärts mit DCS versorgte distale intraartikuläre Femurfraktur. (a) 4 Wochen postoperativ Implantatlockerung mit Instabilität (b) postoperative Röntgen-Kontrolle nach Re-Osteosynthese mit Femur-LISS und Schraubenosteosynthese lat. Kondylus (c) CT distales Femur, frontale Rekonstruktion 4 Monate nach Re-Operation, keine Kallusbildung erkennbar (d) Schraubenbruch und Dislokation des Gelenkblockes nach medial; zwischenzeitlich hatte sich ein erneuter Sturz des Patienten mit der Folge einer Tibiakopffraktur (41 C 2.3) ereignet, mit PlattenZugschraubenosteosynthese (lateral TibiaLISS, medial Kleinfragment-LCP) versorgt (e) Re-Osteosynthese mit Femur-LISS, autologe Spongiosatransplantation, Applikation von Bone Morphogenetic Protein (BMP). 
zeitlich hatte sich der Patient bei einem weiteren Sturz eine Tibiakopffraktur mit metaphysärer Komponente zugezogen, welche lateral mit einem Tibia-LISS und Zugschrauben sowie einer medial abstützenden Locking Compression Plate (LCP) versorgt wurde. Es erfolgte eine Reosteosynthese erneut mit einem Femur-LISS (Abb.2e). Hierbei wurde eine leichte Verkürzung des Femurs in Kauf genommen, um eine gute Einstauchung der Fraktur zu erreichen. Da im diaphysären Knochen kein Auslockerung der winkelstabilen Schrauben zu verzeichnen war, konnten die vorhandenen Schraubenkanäle wieder besetzt werden. Die stark kompromittierten Weichteile verboten einen medialen Zugang und die Implantation eines trikortikalen Beckenkammspanes. Es erfolgte die Applikation von Bone Morphogenetic Protein (BMP) zusammen mit autologer Beckenkammspongiosa über den lateralen Zugang.

\section{Fraktur-Redislokation}

Fraktur-Redislokationen sind zumeist das Ergebnis insuffizienter operativer Versorgung. Mit der Einführung winkelstabiler Implantate für das distale Femur (LISS Less Invasive Stabilisation System, DFN - Distaler Femurnagel [winkelstabil mit Spiralklinge]) sind die technischen Voraussetzungen für zumindest übungsstabile Osteosynthesen gegeben.

Eine besondere Herausforderung stellt die Zunahme periprothetischer Femurfrakturen dar. Zum einen sind die Möglichkeiten der Implantatverankerung durch die Prothese eingeschränkt, zum anderen liegt meistens eine osteoporotische Knochenstruktur vor.

\section{Fallbeispiel 3}

Im vorliegenden Beispiel erfolgte primär auswärts bei einer distalen periprothetischen Femurfraktur (Abb.3a) der insuffiziente Versuch einer Stabilisierung mittels Drahtcerclage (Abb.3b). Nach Fraktur-Redislokation (Abb.3c) wurde eine partiell winkelstabile Osteosynthese mit einer $95^{\circ}$-Kondylenplatte vorgenommen (Abb.3d). Bei ausgeprägter Osteoporose kam es zum Fixationsversagen durch Schraubenausriss im Schaftbereich $(\mathbf{A b b} . \mathbf{3 e}$ ). Daraufhin erfolgte die Versorgung mittels 13-Loch-LIS-System (Abb. 3f) unter Nutzung monokortikaler, periprothetischer Schrauben im Schaftbereich. Nach 12 Monaten zeigt sich eine Konsolidierung der Fraktur (Abb.3g) bei gutem klinischen Ergebnis (Abb.3h).

\section{Postoperative Fehlstellung}

Für die Indikation zur Korrektur postoperativer Achsfehlstellungen gibt es folgende Orientierungsgrößen:

1. Gelenkstufen von mehr als $2 \mathrm{~mm}$ Höhe; 2. Mehr als $10^{\circ}$ Innenrotation; 3. Mehr als $20^{\circ}$ Außenrotation; 4. Mehr als $10^{\circ}$ Varus; 5. Mehr als $15^{\circ}$ Valgus; 6 . Mehr als $10^{\circ}$ Extension oder Flexion. Bei der Indikationsstellung zur Revision müssen selbstverständlich weitere Faktoren berücksichtigt werden wie Lebensalter, Vorerkrankungen, zeitlicher Abstand zur Erstversorgung, Ausmaß des Weichteilschadens, Zusatzverletzungen usw. Die Komplexität auch des Zusammenspiels dieser Faktoren macht die Entscheidung zur Revision immer zu einer Einzelfallentscheidung, welche in ihrer Konsequenz mit dem Patienten eingehend besprochen werden muss.

\section{Fallbeispiel 4}

Im vorliegenden Fall handelt es sich um eine primär auswärts in der dargestellten Art und Weise insuffizient versorgten drittgradig offenen distalen Femurfraktur (Abb.4a). Es liegt eine Fehlstellung in Valgus und Extension, eine starke Verkürzung sowie eine Gelenkstufe vor. In derartigen Defektsituationen hilft die Orientierung am kontralateralen, unverletzten Femur. Bei der präoperativen Planung zeigte sich, dass das anatomisch vorgeformte Femur-LISS bei paralleler Position der distalen (Gelenkblock-)Schrauben zur Gelenkfläche die korrekte Position der Femurachse vorgibt (Abb.4b). Intraoperativ wird die Reposition über einen temporär anmontierten externen AO-Fixateur gehalten (Abb.4c). Im weiteren Verlauf wurde aufgrund des ausgedehnten Defektes die Transplantation eines autologen trikortikalen Beckenkammspanes und autologer Spongiosa durchgeführt (Abb.4d).

\section{Implantatversagen}

Die Ursachen, welche zu Implantatversagen führen, sind vielfältig. Zum einen sind Pseudarthrosen und verzögerte Frakturheilung häufige Ursachen für Ermüdungsbrüche der Implantate im Frakturbereich. Des Weiteren kann das Belassen von Knochendefekten ein Versagen des Implantates verursachen. Und zuletzt kann osteoporotischer Knochen die stabile Verankerung des Implantats erschweren mit der sekundären Konsequenz des Implantatausrisses und Fixationsversagens.
Abb. 3 (Seite 162) 71-jähriger Patient, periprothetische distale Femurfraktur. (a) präoperativ (b) primär auswärts insuffizient mit einer Drahtcerclage versorgt, postoperatives Röntgenbild (c) Frakturredislokation im seitlichen Röntgenbild erkennbar (d) postoperative Röntgenkontrolle nach ebenfalls auswärtigem Zweiteingriff, Osteosynthese mit einer $95^{\circ}$ Kondylenplatte (e) nachfolgend Schraubenausriss diaphysär und Fixationsversagen mit Frakturredislokation; Zuweisung in unsere Klinik (f) postoperative Röntgenkontrolle in 2 Ebenen nach Re-Re-Osteosynthese, Einsatz des 13-Loch-LIS-Systems, Fixation des Implantates diaphysär mit speziellen Schrauben für den periprothetischen Einsatz (abgeflachte Schraubespitze, monokortikal, winkelstabil, selbstschneidend) (g) Röntgenbild 12 Monate nach Fraktur (h) gutes klinisches Ergebnis mit unter Vollbelastung mobilisiertem Patienten.

Die Strategien für das Vorgehen bei Pseudarthrosen und verzögerter Frakturheilung wurden im Abschnitt 2 besprochen. Ist es im Rahmen der Pseudarthrosenentwicklung auch zu einem Implantatversagen gekommen, muss entschieden werden, ob ein Verfahrens- oder Implantatwechsel erforderlich ist. Oben sind die Kriterien zum Verfahrenswechsel oder zur Implantatmodifikation bei intakten Implantaten besprochen worden. Ist das Implanat gebrochen, ausgerissen oder disloziert, muss ein Wechsel von Teilen oder des gesamten Implantates vorgenommen werden.

\section{Fallbeispiel 5}

Ein erneutes Beispiel für eine periprothetische distale Femurfraktur. Bei verzögerter Frakturheilung Bruch der 95Kondylenplatte (Abb.5a). Problematisch bei periprothetischen Femurfrakturen kommt hinzu, dass die oft hochbetagten Patienten aufgrund ihres reduzierten körperlichen Zustandes nicht teilbelasten können und das Implantat daher wiederholt (zyklisch) hohen Belastungen ausgesetzt ist. Das gebrochene Implantat wird entnommen und durch ein FemurLISS ersetzt (Abb.5 b). Wichtig ist bei der Implantation eines LISS in einem solchen Fall, dass das Implantatende über die Spitze des Prothesenschaftes hinausreicht, um eine gute Verteilung der auftretenden Kräfte zu bewirken und keine Stresskonzentration zwischen den Implantatenden zu verursachen. Perioprothetische Femurfrakturen sind durch das Vorhandensein von osteoporotischen Knochen gekennzeichnet. Durch das Besetzen möglichst vieler Schraubenoptionen im distalen Bereich steigt die Kon- 
OP-JOURNAL 2/2004
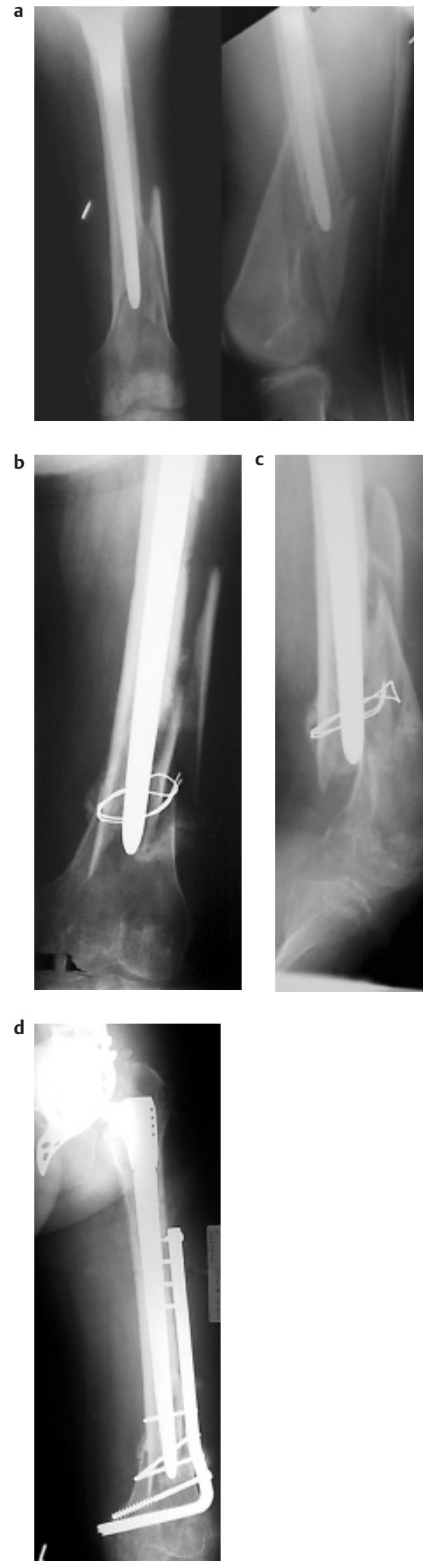

e

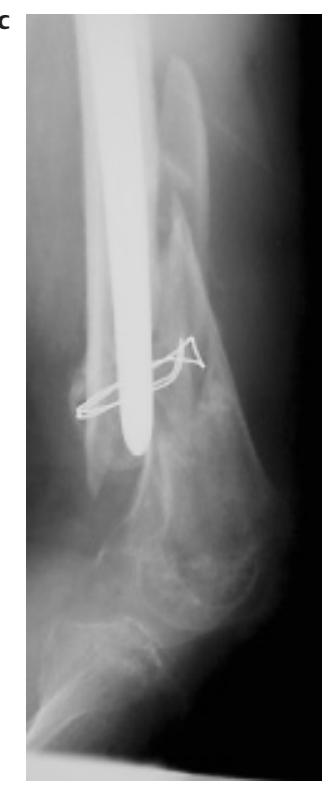

g

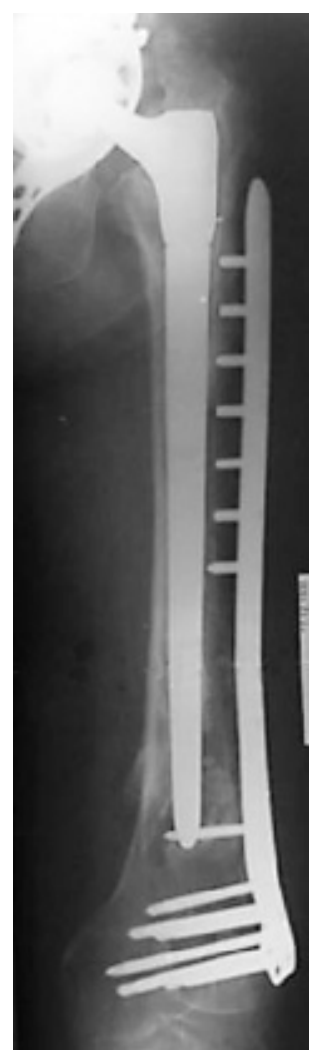

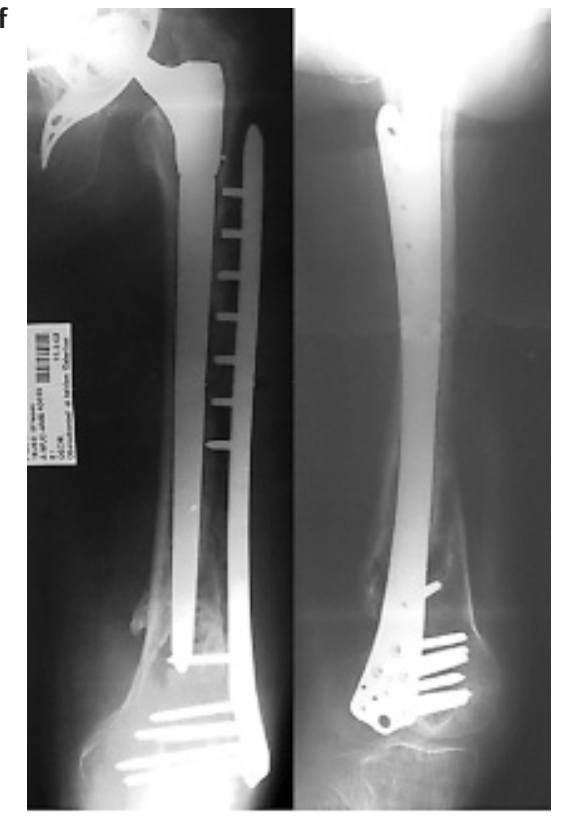

h

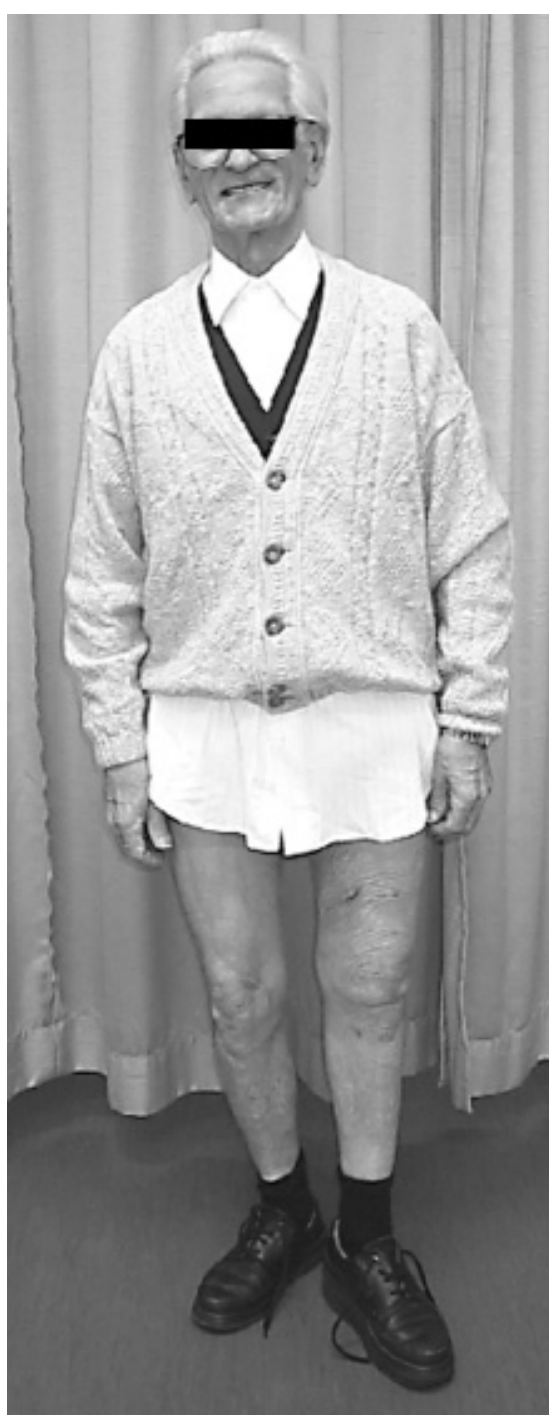

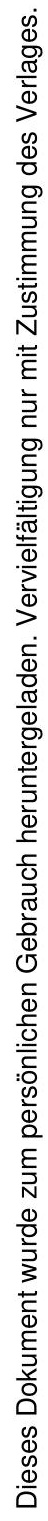




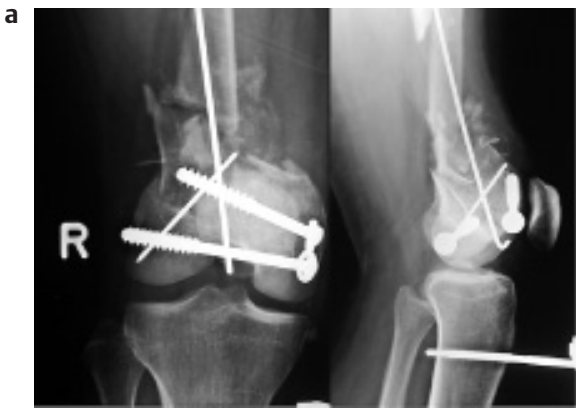

b
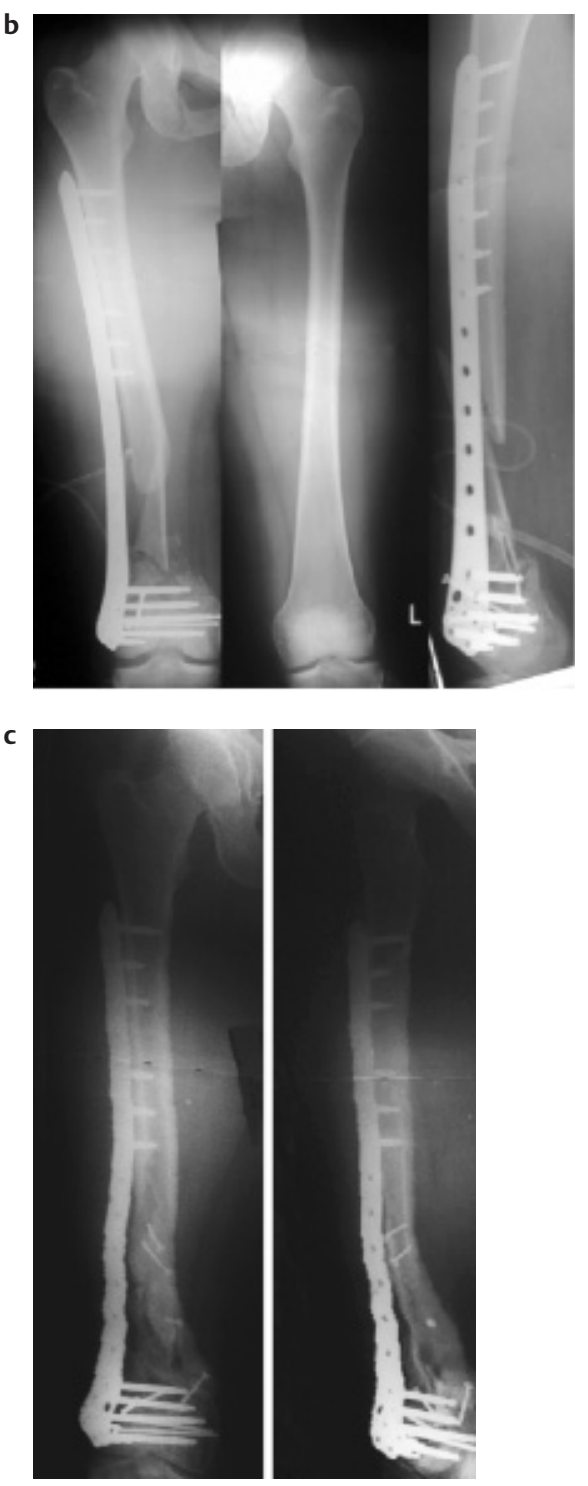

taktfläche zwischen Knochen und Implantat. Somit wird die Belastung des Knochens - insbesondere der Spongiosa - auf eine größere Fläche verteilt und die Gefahr des Auslockerns der Osteosynthese ist geringer [7].

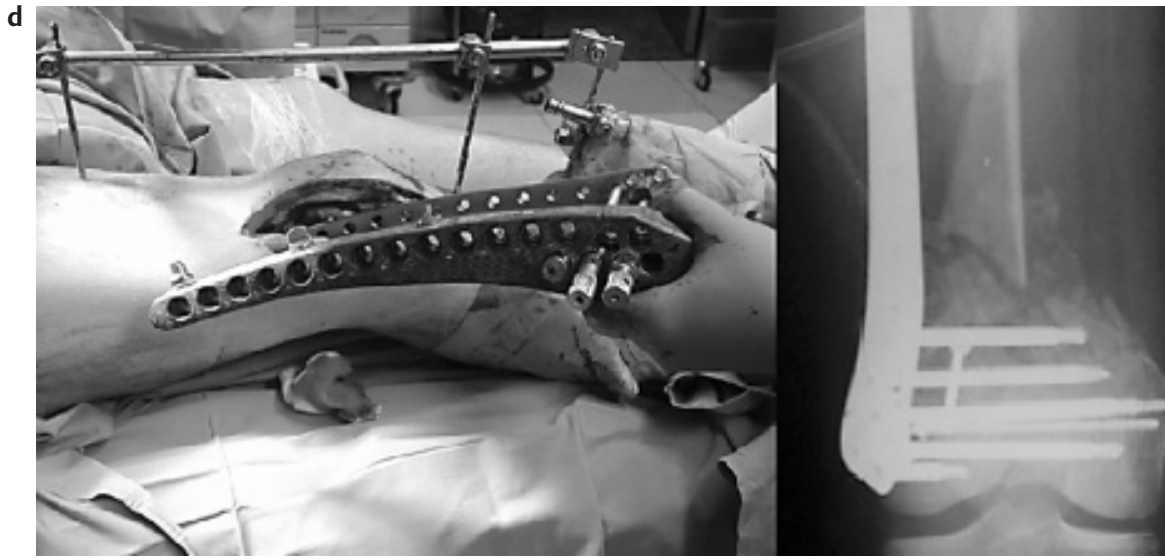

Abb. 4 39-jährige, polytraumatisierte Patientin, III-gradig offene, distale intraartikuläre Femurfraktur mit Knochenverlust. (a) primär auswärts in der dargestellten Art und Weise insuffizient versorgt (b) postoperative Röntgenkontrolle nach Reosteosynthese mit Femur-LISS und Schraubenosteosynthese des lateralen Kondylus; mit dargestellt das kontralaterale intakte Femur welches zur Orientierung bei der Rekonstruktion von Länge und Achse diente (c) intraoperative Darstellung des Einschiebens des LISS mittels Zielbügel; die Reposition der Fraktur wird mittels Fixateur externe gehalten; die Ausrichtung des Gelenkblockes und damit die Positionierung des LISS wird durch die parallel zur distalen femoralen Gelenklinie eingebrachten LISS-Schrauben vorgegeben (entsprechende präoperative Planung nötig) (d) im Verlauf Transplantation eines autologen trikortikalen Beckenkammspanes und autologe Spongisatransplantation analog zu Fallbeispiel 1.

\section{Fallbeispiel 6}

In den vorhergehenden Fallbeispielen wurden die Probleme nach Osteosynthesen am distalen Femur vorwiegend mittels der Applikation des LIS-Systems für das distale Femur adressiert. Wie oben dargestellt kann es bei lang andauernder zyklischer Belastung auch zu Schraubenbruch der LISS-Schrauben kommen (Fallbeispiel 2). Die publizierte Rate des Implantatversagens beim LIS-System liegt zwischen 5 und $10 \%$. Das häufigste Problem stellen jedoch Schraubenausrisse im Schaftbereich bei Fehlplatzierung der monokortikalen, selbstbohrenden und selbstschneidenden Schrauben dar. Wurde das LISS zu weit dorsal oder ventral am Femurschaft angelegt, a

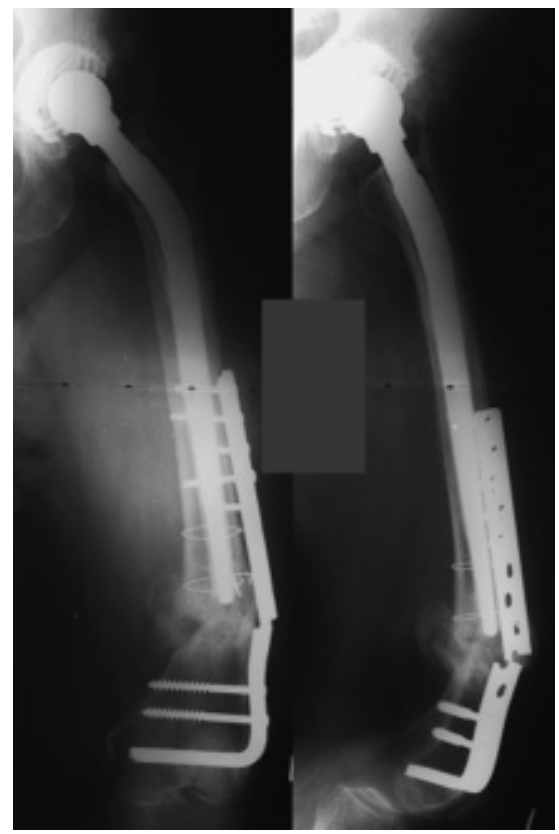

b

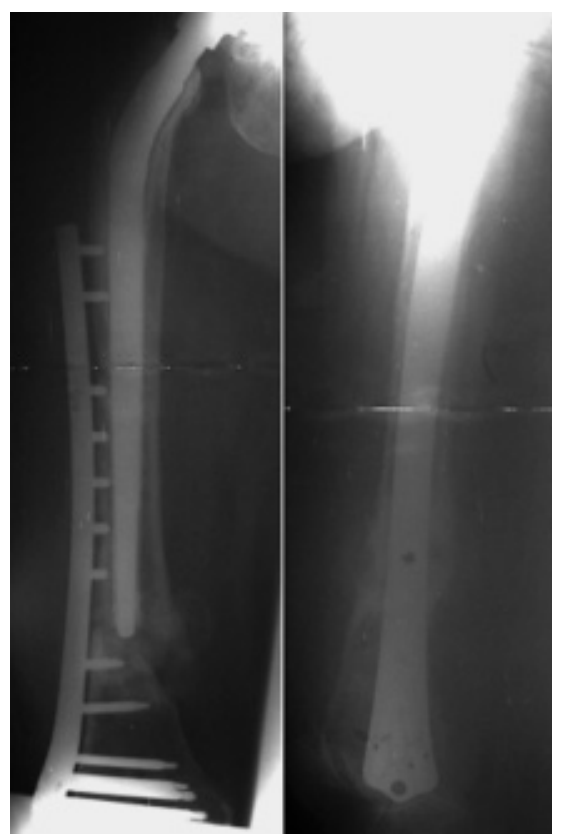

Abb. 5 78-jährige Patientin, periprothetische distale Femurfraktur, auswärts mit $95^{\circ}$ Kondylenplatte versorgt. (a) Ermüdungsbruch des Implantates, es liegen keine Lockerungszeichen der Schrauben vor (b) Re-Osteosynthese mit Femur-LISS. 

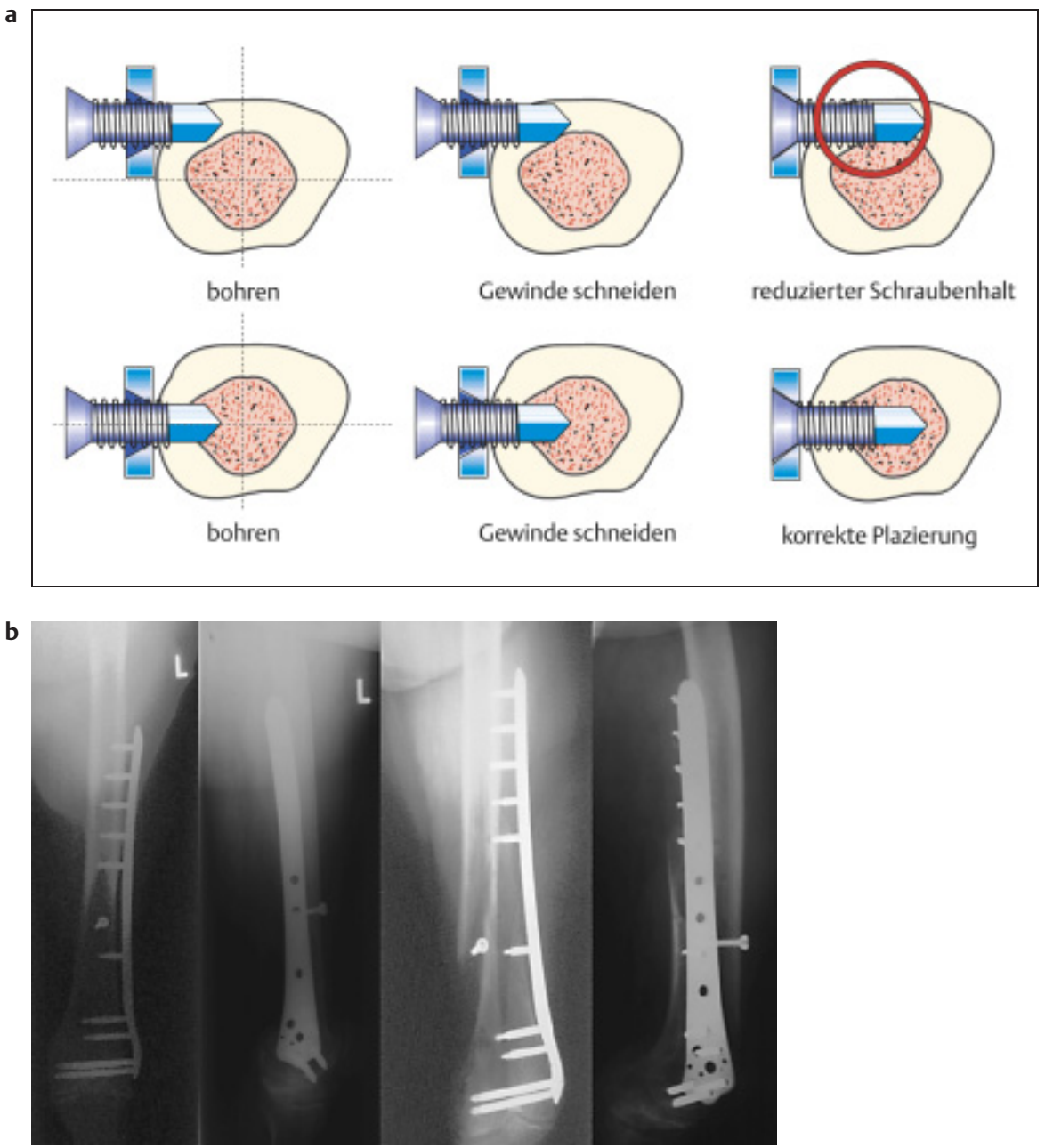

Abb. 6 64-jährige Patientin, Sturz zu ebener Erde, 33 A 2.1. (a) Querschnitt Femurdiaphyse, Einbringen einer winkelstabilen, selbstbohrenden und selbstschneidenden Schraube. Obere Reihe: tangentiales Einbringen der Schraube, dadurch im äußeren Schraubenbereich kein Gewindeschneiden und entsprechend auch keine Schraubenverankerung. Untere Reihe: korrekte, zentrale Schraubenplatzierung (b) links die postoperativen Röntgenbilder in a.p. und lateraler Projektion, rechts die Röntgenkontrolle im Verlauf mit diaphysär ausgerissenem Implantat.

wurden die monokortikalen Schrauben tangential in der Kortikalis platziert und verfügten (Abb.6a) so über eine unzureichende Haltekraft. Im vorliegenden Fall kam es nach primär suffizient erscheinender Osteosynthese zum Ausriss der proximalen monokortikal eingebrachten Schaftschrauben (Abb.6b). In einem solchen Fall kann die Reosteosynthese ebenfalls mit dem LISS erfolgen. Empfehlenswert ist es dann, 2 bis 3 der diaphysären Schrauben bikortikal zu setzen, da in der lateralen Kortikalis der Abstand zwischen den insuffizienten und den neu einzubringenden Bohrkanälen gering ist. Diese Probleme traten vor allem zu Beginn der klinischen Einführung des LISS auf und waren auf die mangelnde Erfahrung der Operateure mit diesem Implantat zurückzuführen, da das Gefühl des Eindrehens der Schrauben sich von konventionellen Implantaten unterschied. Dieses Problem ist aber adressiert und wird durch entsprechende intraoperative Kontrollen vermieden.

\section{Schlussfolgerung}

Auch für die Rekonstruktion distaler Femurfrakturen nach fehlgeschlagenen Osteosynthesen gilt, dass zunächst eine anatomische Rekonstruktion und rigide interne Fixation der Gelenkkomponente der Fraktur erzielt werden muss. Ebenso muss eine stabile Fixation der metaphysären Frakturkomponente unter Berücksichtigung von Länge, Achse und Rotation gewährleistet sein. Diese Ziele sollen unter maximal möglicher Schonung der Durchblutung des Knochens, des Periosts und der Muskulatur erreicht werden.

Dies ist um so wichtiger, da es sich nach fehlgeschlagenen Osteosynthesen am distalen Femur immer mindestens um Sekundäreingriffe handelt und jede zusätzliche Kompromittierung des Gewebes die Chance auf eine Lösung des Problems vermindert.

Hierbei helfen minimalinvasive Operationstechniken, welche keine oder nur eine limitierte Exposition der Fraktur beinhalten. Neue Implantate (LISS, DFN) ermöglichen eine stabile Fixation der metaphsären Frakturkomponente ebenfalls in minimalinvasiver Art und Weise. Speziell bei der extramedullären winkelstabilen Osteosynthese, auf welche sich unsere Erfahrung beim Komplikationsmanagement bei distalen Femurfrakturen vor allem bezieht, haben sich interessante Entwicklungen vollzogen.

Das LISS ist durch seine Konstruktion in der Lage, Weichteile und Durchblutung in idealer Weise zu schonen. Durch die hohe Winkelstabilität kann eine Auflage des Kraftträgers auf dem Periost vermieden und damit die periostale Durchblutung geschont werden. Zusätzlich ermöglicht das LISS durch seinen vorgeformten Kraftträger und den Zielbügel mit dem Trokarsystem das minimalinvasive Einbringen in einfacher Technik. Speziell in Regionen, in denen die Gefäßversorgung kritisch ist, wie dem distalen Femur, zeigt sich die minimalinvasive Technik überlegen. Die ersten Studien zeigten gute Ergebnisse bezüglich der Hauptkomplikationsraten, wie Infektion, Pseudarthrose, Implantatversagen und die Notwendigkeit sekundärer Spongiosaplastiken [9].

Somit haben sich auch die Lösungsmöglichkeiten bei fehlgeschlagenen Osteosynthesen nach unserer Erfahrung durch die Einführung minimalinvasiv einzubringender winkelstabiler Implantate deutlich erweitert. So muss bei schlechten biologischen Verhältnissen eine fehlende Kallusbildung mit fehlender medialer Abstützung am distalen Femur nicht automatisch zum Implantatversagen führen. Es kann somit bei ordnungsgemäß durchgeführter winkelstabiler Osteosynthese auf eine primäre Spongiosaplastik oder die primäre Anlage eines trikortikalen Beckenkammspanes verzichtet werden. Dies kann dann bei ausbleibender Frakturkonsolidierung im Verlauf vorgenommen und muss nicht als „fehlgeschlagene Osteosynthese“ verstanden werden. Vor allem die Konsolidierung der primär unter Umständen erheblich kompromittierten Weichteile kann bei diesem Verfahren abgewartet werden. 
Kommt es nach primärer Versorgung einer distalen Femurfraktur mit einem „konventionellen“ - nicht winkelstabilen - Implantat zum Implantatversagen oder zur Fraktur-Redislokation, scheint uns das Umsteigen auf ein winkelstabiles Implantat indiziert.

Liegt die Ursache für das Implantatversagen zum größten Teil in der mangelhaften Stabilität der Fragmentfixierung begründet, kann die alleinige Reosteosynthese beispielsweise mit einem LISS für den distalen Femur mit gutem Erfolg durchgeführt werden. Liegt eine atrophe knöcherne Situation vor, sind "biologische“ Maßnahmen wie beispielsweise die Transplantation von autologer Spongiosa angezeigt; neuerdings kann auch der Einsatz von Bone Morphogenetic Protein BMP diskutiert werden. Diese osteoinduktiv wirkenden Wachstumsfaktoren (BMP-2 und BMP-7 sind derzeit verfügbar) bewirken eine De-novo-Synthese von Kallus. Die Anwendung dieser Substanzen muss unter gewissen Vorbedingungen und mit speziellen Techniken erfolgen, deren Besprechung an dieser Stelle zu weit führen würde. Ein zusätzlich bestehender, sich nicht durchbauender Knochendefekt sollte mit einem Knochengraft überbrückt werden. Hierbei ist die Transplantation eines autologen trikortikalen Beckenkammspanes zu bevorzugen. Ist die Entnahme eines suffizienten Transplantats nicht möglich, kann auch mit Homograft und zusätzlicher autologer Spongiosa oder BMP-Applikation gearbeitet werden. Kommt es zu einem Versagen des winkelstabilen Implantates, muss auch hier die Analyse der Ursache die Richtung für die weitere Behandlung vorgeben. Liegt die Ursache in den biologischen Rahmenbedingungen begründet, müssen diese durch die oben angeführten Maßnahmen (Spongiosa, Wachstumsfaktoren, Beckenkammspan) verbessert werden. Unserer Erfahrung nach kommt es beim seltenen Implantatversagen des LIS-Systems im diaphysären Bereich zumeist nicht zu Schraubenlocke- rungen am Schrauben/Knochen-Interface. Diese Schraubenlöcher können daher bei der Reosteosynthese eventuell erneut besetzt werden. Im meta- und epiphysären Bereich können bei vorhandener Insuffizienz der Schraubenkanäle durch eine leichte Verkürzung neue Schraubeninsertionsstellen geschaffen werden. Bei älteren Patienten kann auch das Vornehmen einer Verbundosteosynthese und das Auffüllen der Schraubenkanäle mit Knochenzement indiziert sein. Liegt das Implantatversagen beim LIS-System in einer zu rigiden Fixation begründet, hilft bei der Reosteosynthese das längerstreckige Nichtbesetzen von Schraubenoptionen im Frakturbereich und die Verteilung der diaphysären Schrauben auf einen längeren Plattenbereich. Dies bewirkt, dass das Einwirken von Belastungsspitzen auf einen kurzen Bereich des Implantats vermieden wird. Es kann ins solchen Fällen nötig werden, eine entsprechend längeres Implantat $\mathrm{zu}$ verwenden.

Trotz der teilweise hohen Rate an Fehlschlägen bei Osteosynthesen am distalen Femur ist das klinische Ergebnis nach letztendlich erfolgreicher Osteosynthese gut. So zeigen Rademakers et al. in einer aktuellen Studie, dass nach einem Jahr bei der operativen Versorgung intraartikulärer distaler Femurfrakturen zwar nur $62 \%$ radiologisch verheilt waren, dass aber - gemessen an unterschiedlichen Kniescores (HSS und Neer) - im Langzeit-Follow-up (im Mittel 14 Jahre) bei ca. $80 \%$ der Patienten ein gutes bis exzellentes Ergebnis erreicht wird [5]. Dieses Ergebnis sollte in Anbetracht der erweiterten Möglichkeiten des Komplikationsmanagements mittels winkelstabiler, minimalinvasiver Implantate nach osteosynthetischer Versorgung distaler Femurfrakturen optimistisch stimmen: Die frühere „Problemfraktur“ des distalen Femurs könnte sich auch bei ungünstigen Rahmenbedingungen (höhergradiger Weichteilschaden, Osteoporose) zu einer operativ gut behandelbaren Entität wandeln.

\section{Literatur}

${ }^{1}$ Bellabarba C, Ricci WM, Bolhofner BR et al. Indirect reduction and plating of distal femoral nonunions. J Orthop Trauma 2002; 16(5): 287-296

${ }^{2}$ Haas N, Schandelmaier P, Krettek C. et al. Therapeutisches Konzept bei der distalen Femurfraktur mit Gelenkbeteiligung. Hefte Unfallheilkd 1990; 212: 179-187

${ }^{3}$ Johnson EE et al. Combined direct and indirect reduction of comminuted four-part intraarticular T-type fractures of the distal femur. Clin Orthop 1988; 231: 154-162

${ }^{4}$ Krettek C, Schandelmaier P, Richter M, Tscherne H. et al. Distale Femurfrakturen. Swiss Surg 1998; 6: 263-278

${ }^{5}$ Rademakers MV, Kerkhoffs GM, Sierevelt IN Raaymakers EL, Marti RK et al. Intra-articular fractures of the distal femur: a long-term follow-up study of surgically treated patients. J Orthop Trauma 2004; 18(4): 213 219

${ }^{6}$ Sanders R, Regazzoni P, Ruedi TP et al. Treatment of supracondylar-intracondylar fractures of the femur using the dynamic condylar screw. J Orthop Trauma 1989; 3(3): 214-222

${ }^{7}$ Schandelmaier P, Stephan C, Krettek C, Tscherne H. Distal fractures of the femur Unfallchirurg 2000; 103(6): 428-436

${ }^{8}$ Schatzker J, Lambert D. et al. Supracondylar fractures of the femur. Clin Orthop 1979, 138: $77-83$

${ }^{9}$ Schütz M, Müller M, Krettek C, Höntzsch D, Regazzoni P, Ganz R, Haas N et al. Minimally invasive fracture stabilization of distal femoral fractures with the LISS: a prospective multicenter study. Results of a clinical study with special emphasis on difficult cases. Injury 2001; 32 Suppl 3: 48-54

10 Stewart MJ, Sisk TD, Wallce SL et al. Fractures of the distal third of the femur. J Bone Joint Surg 1966; 48-A: 784-807

${ }^{11}$ Tscherne H, Oestern H, Trentz O et al. Spätergebnisse der distalen Femurfraktur und ihre besonderen Probleme. Zentralbl Chir 1977; 102 (15): 897-904

${ }^{12}$ Wang JW, Weng LH et al. Treatment of distal femoral nonunion with internal fixation, cortical allograft struts, and autogenous bone-grafting. J Bone Joint Surg 2003; 85A: $436-440$

\section{Dr. med. Hermann Josef Bail} Oberarzt

Priv.-Doz. Dr. med. Michael Schütz Oberarzt

Prof. Dr. med. Norbert Haas

Direktor

Centrum für Muskuloskeletale

Chirurgie, Klinik für Unfall- und Wiederherstellungschirurgie, Charitè Universitätsmedizin Berlin, Humboldt Universität und Freie Universität Berlin Augustenburger Platz 1

D-13353 Berlin 